\title{
The influence of osteoporosis and cardiometabolic syndrome risk factors on bone mineral density of females with thyroid dysfunction
}

Gluvic Z, Samardzic V, Lackovic M, Tica-Jevtic J, Vujovic M, Popovic-Radinovic V, Mitrovic B, Popin-Taric M, Vasic-Vlaisavljevic A, Isenovic ER.

\section{Introduction}

The effects of TSH and thyroid hormones on bone turnover are well-known. The aim of this study is to examine the influence of osteoporosis $(\mathrm{Op})$ and cardiometabolic syndrome risk factors on bone mineral density (BMD) in the population of age-matched, normotensive and nondiabetic females.

\section{Material and methods}

Cross-sectional study involved 76 females (average age $53 \pm 10$ years), divided into 3 groups: hypo-, hyper-, and euthyroid. Presence of smoking habit, previous fracture/s and menopause were Op risk factors while body mass index (BMI), waist circumference (WC), triglycerides, and cholesterol levels were cardiometabolic risk factors. Index of cardiometabolic burden (ICMB) consisted of BMI $>30 \mathrm{~kg} / \mathrm{m} 2$ and $/ \mathrm{or} \mathrm{WC}>88 \mathrm{~cm}$ and showed increase in cardiovascular disease risk. Lumbar spine (LS) and left hip (LH) T scores represented DEXA measured of BMD. Osteopenia and Op were defined as T scores $<-1$.

\section{Results}

Decreased BMD was detected in $46(60 \%)$ participants. The lowest $\mathrm{T}$ scores were observed in hypothyroid group. Average LS and $\mathrm{LH}$ T scores were -1.1 and -0.75 respectively, and differed between groups (hypo/hyperthyroid vs euthyroid). Significant influences of $\mathrm{BMI}$ and triglycerides on LH T score (Figures 1 and 2) as well as the presence of previous fractures and menopause on LS/LH T scores were detected. The effect of TSH, FT4 and ICMB on BMD was not significant.

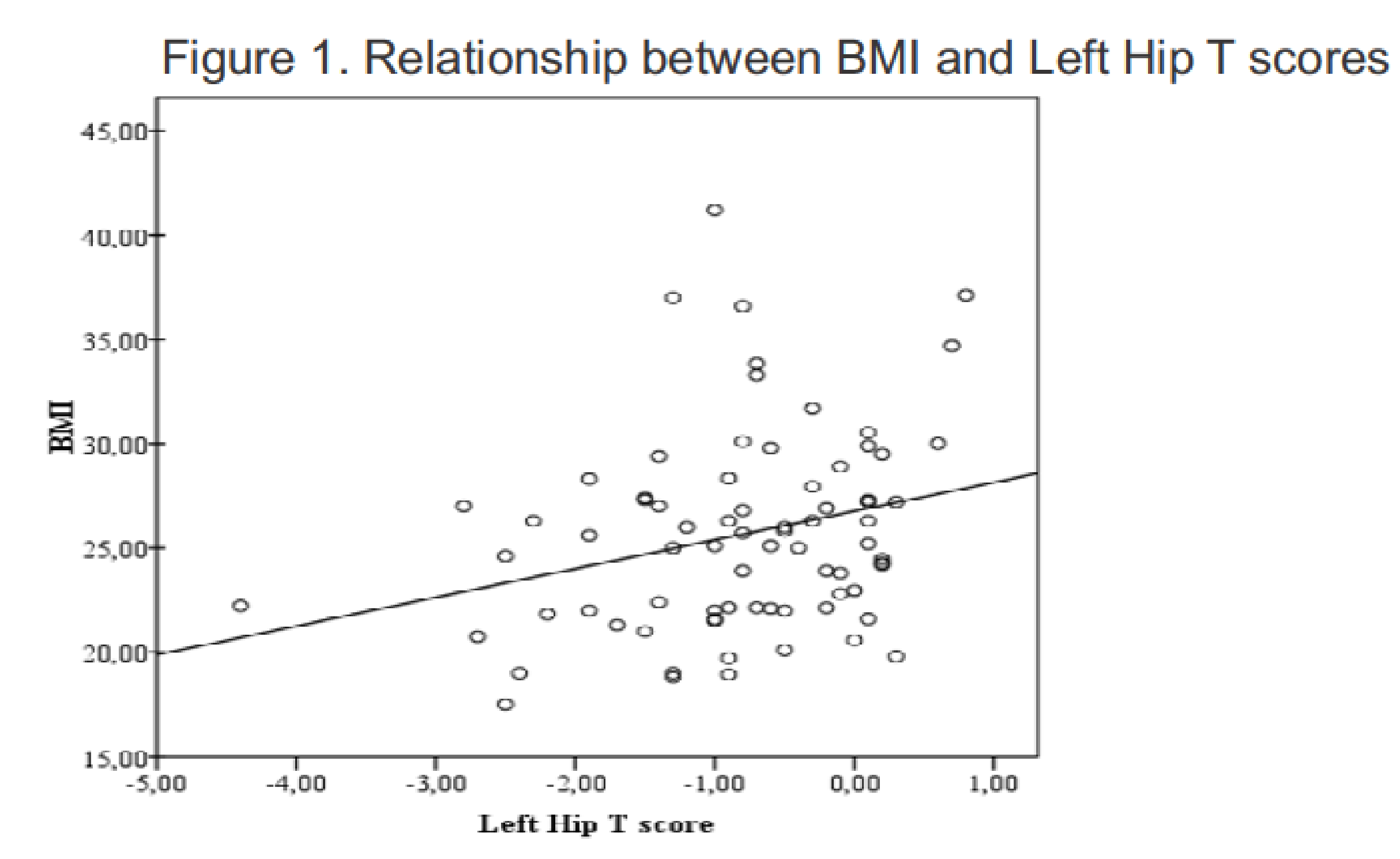

\section{Conclusion}

In the population of females with thyroid dysfunction on initial presentation, osteopenic BMD was registered. The presence of previous fractures and menopause, BMI, and triglycerides significantly affected BMD, while TSH, FT4, and ICMB did not affect BMD.

Key words: bone mineral density, risk factors, thyroid dysfunction 